\title{
Incidencia de la animación a la lectura en la mejora de la competencia escrita: Una investigación con alumnado inmigrante
}

\author{
Teresa Cervera Mata \\ I.E.S. "Río Andarax", Almería \\ María Pilar Núñez Delgado \\ Universidad de Granada
}

Recibido: 22 febrero 2017 / Aceptado: 28 marzo 2017

ISSN: $1697-7467$

\begin{abstract}
RESUMEN: La animación a la lectura de textos literarios es una herramienta metodológica que puede utilizarse con diferentes finalidades en el contexto escolar. Una de ellas es la mejora de la expresión escrita y, en concreto, del dominio de las propiedades textuales. En este trabajo se detallan los resultados de una investigación realizada en la enseñanza secundaria en un contexto de compensación educativa con alumnado inmigrante. Además de otras medidas de apoyo, con estos estudiantes se pusieron en práctica propuestas de escritura creativa vinculadas a las lecturas realizadas como parte de su proceso de aprendizaje del español como segunda lengua.
\end{abstract}

Palabras clave: Animación lectora, expresión escrita, propiedades textuales, inmigración, español como segunda lengua.

Impact of Reading Animation in Improving Written Competence: An Investigation with Immigrant Students

\begin{abstract}
Promotion of reading of literary texts is a methodological tool that can be used for different purposes in the school context. One of them is the improvement of written expression, and, particularly, of the domain of the textual properties. In this work the results of an investigation carried out in the secondary school under a context of educational compensation with immigrant students are outlined. In addition to other measures of support, with this students were put into practice creative writing proposals linked to the readings made as part of their learning process of Spanish as a second language.

Keywords: Promotion of reading, written expression, textual properties, immigration, Spanish as a second language.
\end{abstract}

\section{INTRODUCCIÓN}

La necesidad de cambios metodológicos que promuevan un mejor aprendizaje de las lenguas y de sus literaturas parece indiscutible a la vista de los numerosos estudios que apoyan esta premisa. Sin embargo, la realidad es que la puesta en práctica de estas innovaciones no es un hecho tan general como sería deseable, y se trata más bien de casos aislados, en los que docentes comprometidos se aventuran en un deseo de mejorar sus clases. Una de 
las herramientas que ha conseguido cambios en la enseñanza de la literatura es el taller de animación lectora (Gómez Villalba y Pérez González, 2001; Perdomo, 2005; ÁlvarezÁlvarez y Pascual-Díez, 2013); de aquí que, con el doble objetivo de intentar demostrar que su aplicación en el aula también es beneficiosa para el alumnado inmigrante en su proceso de aprendizaje del español como segunda lengua, y de observar qué aspectos son los más modificados en la expresión escrita tras su puesta en práctica, se haya realizado el presente estudio. La investigación se ha desarrollado en un contexto de compensación educativa con un grupo de alumnos inmigrantes que cursan la educación secundaria obligatoria.

\section{MARCo teórico}

\subsection{La destreza de la expresión escrita}

Escribir es una de las cuatro habilidades o destrezas que componen la comunicación verbal, junto a leer, hablar y escuchar. Escribir y leer forman parte de las habilidades del código escrito, frente a hablar y escuchar, que son las habilidades de código oral. Según su papel en el proceso comunicativo, escribir y hablar son las habilidades productivas, o de expresión, y leer y escuchar las habilidades receptivas, o de comprensión.

La expresión escrita se percibe a través del canal visual, es decir, se percibe a través del sentido de la vista y se reciben todos los signos simultáneamente. La comunicación se produce de forma elaborada, durante un proceso en el cual el escritor puede rectificar y corregir el texto; y es una comunicación diferida en el tiempo y en el espacio. El mensaje que se transmite permanece en el tiempo debido al soporte estable en el que se graban las palabras; la presencia de códigos no verbales es menor que en la lengua oral. Entre el emisor y el receptor no existe interacción inmediata durante la composición del texto, y el contexto en el que el mensaje se produce no es demasiado relevante, aunque sí puede influir en la composición. La escritura consiste, en definitiva, en alcanzar un objetivo comunicativo por medio de la puesta en marcha de un proceso de composición de un texto escrito. Esta intención de comunicación tiene tanta relevancia que a menudo puede cambiar el significado de las palabras (Cassany, 2004: 25).

La relación entre escritura y lectura es evidente, "lectura y escritura son dos actividades de obligada interdependencia" (Piñán, 2008: 108). En esta óptica se sitúa Delmiro Coto (2002 24), quien cree que ambas habilidades deben trabajarse de forma integrada en la enseñanza de la literatura, pues la lectura es la clave para cimentar el nivel de la lengua que permite la automatización de las reglas básicas de la ortografía, la morfología, la sintaxis y el léxico, dándole alas al lector y al escritor para centrarse en el contenido y la interpretación. En esta línea, Hernández Guerrero afirma que "uno de los principios básicos en los que apoyamos el proceso de aprendizaje es que el escritor ha de ser un lector: escribir es una manera profunda -crítica y placentera- de leer los sucesos de la vida, los escritos de los demás y nuestros propios textos" (2005: 126). Sin embargo, también hay quien plantea la escritura como una forma de evaluar la lectura, porque la escritura es el lugar de las representaciones que vienen a su vez de las lecturas previas, el espacio donde convergen los textos que han sido disfrutados por quien escribe (Acosta, Rodríguez, Flórez y Jurado, 2013: 11, 78). Desde otro ángulo se puede definir la escritura como una actividad 
metalingüística, tal y como señalaba Ong (1982), por la reflexión que conlleva el proceso de escritura en sí mismo, de aquí que la escritura lleve a una buena lectura, ya que el alumno que es capaz de expresarse correctamente a través de un texto escrito, leerá mejor que el que no lo hace, porque, según Moreno (2007), cuando escribimos tomamos conciencia de una serie de dimensiones lingüísticas y discursivas que no están tan presentes cuando leemos. Es decir, la complementariedad de ambas destrezas repercute en la mejora de la competencia comunicativa en la L2. A esto ha de unirse como sustento teórico de nuestra investigación la afirmación de Coseriu (1987) de que los textos literarios tienen una indudable cualidad para la enseñanza de las lenguas dado que en el lenguaje literario cabe la plena potencialidad de las posibilidades de una lengua: variedad de registros, de géneros discursivos, de recursos expresivos, etc.

\subsection{El proceso de escritura}

La enseñanza de la escritura se puede separar en dos ejes: la enseñanza de las características de la escritura, de sus funciones y de las propiedades textuales, por un lado, y de las fases del proceso de composición de textos escritos, por otro. Esta investigación se ha centrado en la enseñanza de la composición escrita atendiendo a las propiedades textuales que Núñez, Fernández de Haro y Romero (2009) utilizaron como variables en su estudio sobre las habilidades metalingüísticas en Educación Primaria. Estas variables se han operativizado siguiendo el modelo propuesto por Rodríguez Polo (1999):

- Coherencia: contenido de la información necesaria, sin excesos ni lagunas; orden de la exposición de las ideas; dominio de la estructura del texto con correcta disposición de los párrafos.

- Cohesión: dominio del uso de campos léxicos, uso adecuado de las figuras de repetición (anáfora en sus distintas formas), utilización de deícticos espaciales y temporales, utilización de enlaces, conectores y marcadores textuales y uso de los signos de puntuación.

- Corrección: corrección morfosintáctica: construcción oracional; corrección léxica y precisión en su uso; corrección ortográfica: ortografía literal y acentuación.

- Adecuación: discurso adecuado al objeto representado, apropiado respecto al destinatario y oportuno respecto a la situación; claridad de propósito(s) y objetivo(s) del texto; mantenimiento del nivel de formalidad en todo el texto.

- Presentación: legibilidad: caligrafía o dominio del trazado; limpieza (ausencia de borrones, enmiendas, tachaduras...); correlación entre espacios en blanco y texto (formato, márgenes, título, espaciado interlineal).

- Estilo: variedad y riqueza léxica, complejidad y variación sintáctica, creatividad expresiva.

Estas propiedades textuales así definidas en un nivel de competencia bastante elemental son las que constituyen las variables dependientes relativas a la expresión escrita utilizadas en el estudio de la presente investigación por tratarse de alumnado para el que el español no es su lengua materna. 


\section{Diseño Y MÉtodo}

Tras la revisión bibliográfica relacionada con la expresión escrita y con los talleres de animación lectora, se aborda la de estudios orientados a comprobar el efecto de la segunda sobre la primera, como los de Perdomo (2005) y Gómez Villalba (1994, 2000, 2001, 2007). Sin embargo, no se han encontrado estudios precedentes que relacionaran la aplicación de los talleres de animación lectora con la mejora de la expresión escrita en contextos de compensación educativa con alumnado inmigrante que está aprendiendo el español como segunda lengua.

Por este motivo se optó por un estudio de carácter exploratorio, cuasi-experimental, deductivo, con un diseño longitudinal, cuantitativo y de seguimiento. La investigación consistió en el desarrollo de un programa compuesto por nueve talleres de animación lectora en un grupo de tercero de ESO conformado únicamente por alumnado inmigrante de origen marroquí, en un centro de compensación educativa de Almería capital. Tras la realización de los talleres se procedió a comprobar si estos tenían repercusión en la expresión escrita de los sujetos. Para ello se compararon los resultados obtenidos en el ejercicio de expresión escrita realizado antes y después de los talleres de animación lectora (pretest-postest).

\subsection{Variables utilizadas}

\subsubsection{Variables intervinientes}

Las variables intervinientes se recogieron mediante un cuestionario que nos proporcionó los siguientes datos sobre los sujetos estudiados:

Tabla 1: Relación y datos de las variables intervinientes.

\begin{tabular}{|c|c|c|c|}
\hline \multicolumn{2}{|c|}{ Variables intervinientes } & $\begin{array}{c}\text { Número de } \\
\text { participantes }\end{array}$ & Porcentaje \\
\hline \multirow{3}{*}{ Edad } & 14 años & 0 & $0 \%$ \\
\cline { 2 - 4 } & 15 años & 6 & $66,6 \%$ \\
\cline { 2 - 4 } & 16 años & 1 & $11,1 \%$ \\
\cline { 2 - 4 } & 17 años & 2 & $22,2 \%$ \\
\hline \multirow{3}{*}{ Sexo } & Hombre & 6 & $66,6 \%$ \\
\cline { 2 - 4 } & Mujer & 3 & $33,3 \%$ \\
\hline \multirow{2}{*}{ Lengua materna } & España & 9 & $0 \%$ \\
\cline { 2 - 4 } & Marruecos & 0 & $100 \%$ \\
\hline & Español & 9 & $0 \%$ \\
\hline
\end{tabular}




\begin{tabular}{|c|c|c|c|}
\hline \multirow{3}{*}{$\begin{array}{c}\text { Lengua del entorno } \\
\text { familiar }\end{array}$} & Español & 0 & $0 \%$ \\
\hline & Árabe & 9 & $100 \%$ \\
\hline & Español y árabe & 0 & $0 \%$ \\
\hline \multirow{3}{*}{$\begin{array}{l}\text { Lengua del entorno } \\
\text { escolar }\end{array}$} & Español & 5 & $55,5 \%$ \\
\hline & Árabe & 0 & $0 \%$ \\
\hline & Español y árabe & 4 & $44,4 \%$ \\
\hline \multirow{2}{*}{$\begin{array}{c}\text { Condición de repeti- } \\
\text { dor }\end{array}$} & Sí & 9 & $100 \%$ \\
\hline & No & 0 & $0 \%$ \\
\hline \multirow{3}{*}{ Edad de la madre } & $30-40$ & 6 & $66,6 \%$ \\
\hline & $40-50$ & 3 & $33,3 \%$ \\
\hline & $50-60$ & 0 & $0 \%$ \\
\hline \multirow{3}{*}{$\begin{array}{l}\text { Instrucción de la } \\
\text { madre }\end{array}$} & No tiene estudios & 6 & $66,6 \%$ \\
\hline & Educación básica & 1 & $11,1 \%$ \\
\hline & Bachillerato o FP & 2 & $22,2 \%$ \\
\hline \multirow{2}{*}{ Trabajo de la madre } & Trabajo manual & 5 & $55,5 \%$ \\
\hline & Sin trabajo & 4 & $44,4 \%$ \\
\hline \multirow{3}{*}{ Edad del padre } & $30-40$ & 3 & $33,3 \%$ \\
\hline & $40-50$ & 4 & $44,4 \%$ \\
\hline & $50-60$ & 2 & $22,2 \%$ \\
\hline \multirow{3}{*}{ Instrucción del padre } & No tiene estudios & 5 & $55,5 \%$ \\
\hline & Educación básica & 4 & $44,4 \%$ \\
\hline & Bachiller o FP & 0 & $0 \%$ \\
\hline
\end{tabular}




\begin{tabular}{|c|c|c|c|}
\hline \multirow{2}{*}{ Trabajo del padre } & Trabajo manual & 6 & $66,6 \%$ \\
\hline & Sin trabajo & 3 & $33,3 \%$ \\
\hline \multirow{3}{*}{$\begin{array}{c}\text { Número de lectores en } \\
\text { la familia }\end{array}$} & 1 & 4 & $44,4 \%$ \\
\hline & 2 & 3 & $33,3 \%$ \\
\hline & 3 & 2 & $22,2 \%$ \\
\hline \multirow{4}{*}{$\begin{array}{l}\text { Lecturas del ámbito } \\
\text { familiar }\end{array}$} & Nada & 0 & $0 \%$ \\
\hline & Libros & 3 & $33,3 \%$ \\
\hline & Libros y periódicos o revistas. & 4 & $44,4 \%$ \\
\hline & Periódicos y revistas & 2 & $22,2 \%$ \\
\hline \multirow{5}{*}{$\begin{array}{l}\text { Libros del ámbito } \\
\quad \text { familiar }\end{array}$} & $\begin{array}{l}\text { Obras literarias de género } \\
\text { narrativo, lírico o teatral. }\end{array}$ & 2 & $22,2 \%$ \\
\hline & Enciclopedias y libros de consulta & 11 & $11,1 \%$ \\
\hline & Libros de texto & 1 & $11,1 \%$ \\
\hline & Libros de cocina, bricolaje & 0 & $0 \%$ \\
\hline & Ninguno & 5 & $55,5 \%$ \\
\hline \multirow{3}{*}{$\begin{array}{l}\text { Profesión a la que } \\
\text { aspira en el futuro }\end{array}$} & Manual & 8 & $88,8 \%$ \\
\hline & Intelectual & 1 & $11,1 \%$ \\
\hline & Artístico & 0 & $0 \%$ \\
\hline \multirow{5}{*}{$\begin{array}{c}\text { Vía de comunicación } \\
\text { social }\end{array}$} & Carta & 0 & $0 \%$ \\
\hline & Cara a cara & 0 & $0 \%$ \\
\hline & Email & 0 & $0 \%$ \\
\hline & Teléfono & 0 & $0 \%$ \\
\hline & Messenger, tuenti, facebook & 9 & $100 \%$ \\
\hline \multirow{5}{*}{$\begin{array}{l}\text { Valoración personal } \\
\text { del uso del español }\end{array}$} & Muy bueno & 8 & $88,8 \%$ \\
\hline & Bueno & 0 & $0 \%$ \\
\hline & Regular & 1 & $11,1 \%$ \\
\hline & Malo & 0 & $0 \%$ \\
\hline & Muy malo & 0 & $0 \%$ \\
\hline
\end{tabular}




\subsubsection{Variables dependientes}

En cuanto a las variables dependientes, fueron elaboradas a partir de las que definen Cassany, Luna y Sanz (1997) y de las que utilizan en su estudio de 2008 Núñez, Fernández y Romero: Coherencia, Cohesión, Corrección, Adecuación, Presentación y Estilo. Para medirlas se elaboró una escala de observación de la composición escrita que fue aplicada a los diferentes ejercicios elaborados por los sujetos del estudio. La escala es regresiva, de forma que el número 5 de la propiedad expresa el grado máximo en el que esta puede presentarse en el texto, y el 1, el grado mínimo en el que aparece.

\subsubsection{Variables independientes}

Por último, las variables independientes que se esperaba que modificaran las variables dependientes de nuestro estudio fueron los nueve talleres de animación lectora que se realizaron en el grupo durante el curso escolar. Cada uno de los talleres correspondía a la lectura de una obra literaria, de género narrativo, lírico o dramático, y se caracterizaba porque sus actividades, además de poseer un fuerte componente lúdico para que resultasen motivadoras, incluían en todos los casos propuestas de escritura creativa diseñadas para la intentar mejorar la expresión escrita.

\subsection{Contexto del estudio}

La investigación se realizó a lo largo de un curso escolar en un instituto de educación secundaria situado en el barrio almeriense de El Puche, declarado por la administración autonómica andaluza como Zona Necesitada de Transformación Social. La Junta de Andalucía, en el documento Intervención coordinada de la Junta de Andalucía en zonas con necesidades de transformación social (2006), define este tipo de zonas como el espacio urbano claramente delimitado, en cuya población concurren situaciones estructurales de pobreza grave y marginación social, y en el que son apreciables problemas en materias como vivienda, escolarización, empleo, integración social, higiene y salud.

Este barrio se localiza en el extrarradio de la zona este de Almería. Fue construido para realojar a la población de los barrios más humildes de la ciudad afectados por las lluvias torrenciales de 1970. Situado en plena terraza fluvial, entre el río Andarax, la vía del ferrocarril Almería-Linares-Baeza-Madrid y la carretera N-334 Almería-Níjar, el diseño del barrio lo condenó a la segregación espacial con respecto al resto de la ciudad. Tiene una superficie de veintiuna hectáreas y alberga una población de unos 5.500 habitantes, aunque se estima que la cantidad de habitantes puede ascender hasta 9.000. Este dato es difícil de concretar debido a la gran movilidad de la población.

La inmigración comienza a crecer en el barrio a partir del año 2000, llegando a albergar más de veinte nacionalidades diferentes. La población marroquí, con un $49 \%$ del total del barrio, se ha convertido en la etnia mayoritaria, desplazando a la población gitana que ha pasado del $56 \%$ al $24 \%$; si bien es cierto que estos datos fluctúan continuamente debido a la continua movilidad de la población. 
Existe entre las familias de El Puche un alto grado de desestructuración y una gran proliferación de graves problemas sociales. Dentro del barrio podemos distinguir dos amplios grupos con características bien diferenciadas: el alumnado de etnia gitana y el alumnado marroquí. En este último grupo hemos basado la presente investigación. Presentan características sociodemográficas como las siguientes:

- Las cargas familiares del alumnado dificultan su normal escolarización.

- Los recursos económicos de las familias son muy escasos.

El nivel cultural es bajo y su dominio de la lengua de instrucción es escaso. El árabe lo emplean únicamente de forma oral, nunca escrita.

- El uso del español se restringe exclusivamente al ámbito escolar.

- Todo esto genera unas dificultades en el proceso de enseñanza-aprendizaje que se concretan en aspectos como un desfase curricular generalizado, ausencia de hábitos de estudio, graves deficiencias en las habilidades instrumentales básicas, escasa motivación hacia el aprendizaje, ausencia de confianza en sus posibilidades de éxito, falta de equilibrio emocional y carencias en las expectativas de futuro.

El centro no está dotado con los recursos tecnológicos suficientes para poder incluir las TIC en la práctica docente, hecho que, aunque en un principio pueda limitar el proceso de enseñanza-aprendizaje con las consecuencias ya conocidas (Núñez y Liébana, 2004), sirve de estímulo para superar esta dificultad, tal y como indica Ramos García (2010), en este caso a través de los talleres de animación lectora.

\subsection{Estudios estadísticos realizados}

Se han utilizado diferentes estudios estadísticos, realizados para poder interpretar posteriormente los datos de la presente investigación de corte cuantitativo: el estudio descriptivo de la distribución de las frecuencias, el test chi-cuadrado de homogeneidad entre grupos, así como las medidas de tendencia central y de dispersión.

La distribución de las frecuencias es una ordenación en forma de tabla de los datos estadísticos en la que se asigna a cada dato su frecuencia correspondiente. De esta forma se puede mostrar los datos de cada variable y su frecuencia en ambos grupos, el control y el experimental. La distribución de las frecuencias informa sobre el valor que adopta una variable y sobre el número de veces que se repite cada uno de estos valores.

La prueba del chi-cuadrado de Pearson se utiliza para analizar la dependencia o independencia entre dos variables cualitativas, y poder así determinar si existe relación entre estas. El p-valor es la probabilidad de obtener un estadístico tan grande o más como el que ha salido, si la hipótesis nula fuera cierta. En este caso, la hipótesis nula es una hipótesis de homogeneidad; eso quiere decir que la variable que estamos probando se distribuye igual en el grupo de control que en el grupo experimental. Puesto que el p-valor es una probabilidad, su valor numérico oscilará entre el 0 y el 1. En este caso concreto, la interpretación de los valores del p-valor es la siguiente: cuando el p-valor es inferior a 0,05 diremos que existen diferencias significativas entre el pretest y el postest. 
Se han estudiado las medidas de tendencia central, media y moda, y las de dispersión, varianza y desviación típica. Dentro del estudio de las medidas de tendencia central, la investigación ha destacado la importancia de la moda para la reflexión acerca del comportamiento de los sujetos. También se ha estudiado la media aritmética que consiste en la suma de los datos dividida por el número total de observaciones (Fernández y Fuentes, 1995: 88). En las medidas de dispersión se ha procedido al cálculo de la varianza que consiste en "la media aritmética de los cuadrados de las desviaciones de los datos respecto a la media" (Fernández y Fuentes, 1995: 145); este cálculo es necesario para conseguir la desviación típica, pues se trata de la raíz cuadrada de la varianza que se utilizará para verificar si la media obtenida es significativa o no.

\section{Análisis de los resultados obtenidos}

Tras la realización de los ejercicios de expresión escrita, al inicio y al final del curso escolar, se efectuaron los estudios estadísticos indicados en el apartado anterior, que dieron como resultados los datos que se reflejan en la siguiente tabla y que se comentan a continuación:

Tabla 2: Relación de resultados obtenidos para cada una de las variables dependientes de la expresión escrita en el pretest y en el postest.

\begin{tabular}{|c|c|c|c|c|c|c|c|c|c|c|c|c|}
\hline Variables & \multicolumn{3}{|c|}{$\begin{array}{l}\text { Distribución de } \\
\text { las frecuencias }\end{array}$} & p-valor & \multicolumn{2}{|c|}{ Media } & \multicolumn{2}{|c|}{ Mediana } & \multicolumn{2}{|c|}{ Moda } & \multicolumn{2}{|c|}{$\begin{array}{l}\text { Desviación } \\
\text { típica }\end{array}$} \\
\hline \multirow{6}{*}{ Coherencia } & $\mathrm{P}$. & Pre & Post & \multirow{6}{*}{0,199} & \multirow{3}{*}{ Pre } & \multirow{3}{*}{ Post } & \multirow{3}{*}{ Pre } & \multirow{3}{*}{ Post } & \multirow{3}{*}{ Pre } & \multirow{3}{*}{ Post } & \multirow{3}{*}{ Pre } & \multirow{3}{*}{ Post } \\
\hline & 1 & 3 & 0 & & & & & & & & & \\
\hline & 2 & 4 & 4 & & & & & & & & & \\
\hline & 3 & 2 & 2 & & \multirow{3}{*}{1.89} & \multirow{3}{*}{3.00} & \multirow{3}{*}{2} & \multirow{3}{*}{3} & \multirow{3}{*}{2} & \multirow{3}{*}{2} & \multirow{3}{*}{0.78} & \multirow{3}{*}{1.12} \\
\hline & 4 & 0 & 2 & & & & & & & & & \\
\hline & 5 & 0 & 1 & & & & & & & & & \\
\hline \multirow{6}{*}{ Cohesión } & P. & Pre & Post & \multirow{6}{*}{0,017} & \multirow{3}{*}{ Pre } & \multirow{3}{*}{ Post } & \multirow{3}{*}{ Pre } & \multirow{3}{*}{ Post } & \multirow{3}{*}{ Pre } & \multirow{3}{*}{ Post } & \multirow{3}{*}{ Pre } & \multirow{3}{*}{ Post } \\
\hline & 1 & 7 & 1 & & & & & & & & & \\
\hline & 2 & 1 & 5 & & & & & & & & & \\
\hline & 3 & 1 & 3 & & & & & & & & & \\
\hline & 4 & 0 & 0 & & 1.33 & 2.22 & 1 & 2 & 1 & 2 & 0.71 & 0.67 \\
\hline & 5 & 0 & 0 & & & & & & & & & \\
\hline & $\mathrm{P}$. & Pre & Post & & & & & & & & & \\
\hline & 1 & 7 & 6 & & Pre & Post & Pre & Post & Pre & Post & Pre & Post \\
\hline & 2 & 2 & 3 & & & & & & & & & \\
\hline Correccion & 3 & 0 & 0 & 1 & & & & & & & & \\
\hline & 4 & 0 & 0 & & 1.22 & 1.33 & 1 & 1 & 1 & 1 & 0.44 & 0.50 \\
\hline & 5 & 0 & 0 & & & & & & & & & \\
\hline
\end{tabular}




\begin{tabular}{|c|c|c|c|c|c|c|c|c|c|c|c|c|}
\hline \multirow{6}{*}{ Adecuación } & P. & Pre & Post & \multirow{6}{*}{0,106} & \multirow{3}{*}{ Pre } & \multirow{3}{*}{ Post } & \multirow{3}{*}{ Pre } & \multirow{3}{*}{ Post } & \multirow{3}{*}{ Pre } & \multirow{3}{*}{ Post } & \multirow{3}{*}{ Pre } & \multirow{3}{*}{ Post } \\
\hline & 1 & 2 & 1 & & & & & & & & & \\
\hline & 2 & 3 & 1 & & & & & & & & & \\
\hline & 3 & 2 & 0 & & \multirow{3}{*}{2.67} & \multirow{3}{*}{4.22} & \multirow{3}{*}{2} & \multirow{3}{*}{5} & \multirow{3}{*}{2} & \multirow{3}{*}{5} & \multirow{3}{*}{1.50} & \multirow{3}{*}{1.56} \\
\hline & 4 & 0 & 0 & & & & & & & & & \\
\hline & 5 & 2 & 7 & & & & & & & & & \\
\hline \multirow{6}{*}{ Presentación } & P. & Pre & Post & \multirow{6}{*}{0,539} & \multirow{3}{*}{ Pre } & \multirow{3}{*}{ Post } & \multirow{3}{*}{ Pre } & \multirow{3}{*}{ Post } & \multirow{3}{*}{ Pre } & \multirow{3}{*}{ Post } & \multirow{3}{*}{ Pre } & \multirow{3}{*}{ Post } \\
\hline & 1 & 1 & 0 & & & & & & & & & \\
\hline & 2 & 1 & 1 & & & & & & & & & \\
\hline & 3 & 5 & 4 & & \multirow{3}{*}{2.89} & \multirow{3}{*}{3.56} & \multirow{3}{*}{3} & \multirow{3}{*}{3} & \multirow{3}{*}{3} & \multirow{3}{*}{4} & & \\
\hline & 4 & 2 & 2 & & & & & & & & 0.93 & 1.01 \\
\hline & 5 & 0 & 2 & & & & & & & & & \\
\hline & P. & Pre & Post & & & & & & & & & \\
\hline & 1 & 9 & 9 & & Pre & Post & Pre & Post & Pre & Post & Pre & Post \\
\hline & 2 & 0 & 0 & & & & & & & & & \\
\hline Estilo & 3 & 0 & 0 & 1 & & & & & & & & \\
\hline & 4 & 0 & 0 & & 1 & 1 & 1 & 1 & 1 & 1 & 0 & 0 \\
\hline & 5 & 0 & 0 & & & & & & & & & \\
\hline
\end{tabular}

a. Coherencia. El p-valor de esta variable es de 0,199, por lo que no constituye un dato significativo, ya que denota que no existen comportamientos diferentes entre el ejercicio inicial y el final. Por lo tanto, la hipótesis nula es una hipótesis de homogeneidad, es decir, la variable Coherencia se distribuye igual antes de la implantación de los talleres de animación lectora que después de su realización.

Si observamos la distribución de las frecuencias y las medidas de tendencia central y dispersión, podemos interpretar que, aunque no de forma significativa, se ha producido un ligero incremento en la variable de la Coherencia en el ejercicio final con respecto al inicial. Se percibe cómo las opciones 4 y 5 , que son las que mejor valoran la coherencia en la expresión escrita, no figuran en el ejercicio inicial, aunque sí en el final. A pesar de no ser estadísticamente significativos, estos datos deben tenerse en cuenta debido a que la muestra de nuestro estudio es reducida y a que se produce una leve mejoría en la variable.

Esto mismo se observa en las medidas de tendencia central, pues la media asciende de un 2 a un 3, aunque la moda sigue anclada en el 2. El hecho de que la desviación típica ascienda en el ejercicio final con respecto al inicial puede interpretarse como una progresión hacia la mejora de la coherencia, pero solo en algunos alumnos del grupo experimental, al no ser un cambio generalizado la desviación típica presenta valores dispersos más alejados de la media que en el ejercicio inicial.

b. Cohesión. En este caso, el p-valor es de 0,017, hecho que sí constituye un dato con significación estadística, ya que denota que existen comportamientos diferentes entre el pretest y el postest. No estamos ante una hipótesis de homogeneidad, esto indica que 
el resultado del ejercicio final ha variado con respecto al inicial en sentido positivo. Apoyándonos en las medidas de tendencia central y de dispersión, se observa una progresión de los resultados, pues en el ejercicio inicial la moda era 1, mientras que en el final es de 2 . Se observa también un descenso en el ejercicio final de la opción 1, la opción con la puntuación más baja posible en el ejercicio, aumentando la frecuencia de las opciones 2 y 3 , y ascendiendo la media de 1,33 a 2.22. En esta variable se observa una desviación típica menor en el ejercicio final que en el inicial, lo que indica una mayor concentración de los datos alrededor de la media.

c. Corrección. El p-valor de la variable Corrección es de 1, esto no es significativo, pues denota que no existen comportamientos diferentes entre el ejercicio inicial y el final. En este caso, la hipótesis nula es una hipótesis de homogeneidad, es decir, la variable Corrección en la expresión escrita se distribuye igual antes de la implantación de los talleres de animación lectora que después de su realización.

Tras la observación de la distribución de las frecuencias, de las medidas de tendencia central y de dispersión se puede afirmar que no se aprecian grandes diferencias entre los resultados obtenidos en el pretest y en el postest en la variable Corrección. La media varía unos puntos, y pasa de 1,22 a 1,33, por lo tanto, la desviación típica se modifica en unas centésimas, de 0,44 a 0,50, y la moda permanece en 1. Aun así, los ligeros cambios que se producen se interpretan como una ligera mejoría en la variable, pues un alumno mejora esta variable en un punto.

d. Adecuación. Para esta variable, el p-valor es de 0,106, por lo que tampoco constituye un dato significativo desde el punto de vista estadístico, indicando que no existen comportamientos diferentes entre ambos ejercicios. La variable Adecuación se distribuye igual antes de la implantación de los talleres de animación lectora que después de su realización, es decir, la hipótesis nula es una hipótesis de homogeneidad.

Aunque el estadístico obtenido en el p-valor no sea significativo como para afirmar que existe una clara progresión en la variable Adecuación, podemos interpretar, teniendo en cuenta el tamaño de nuestra muestra, que sí se ha producido un ligero cambio, pues todas las opciones disminuyen en el ejercicio final, excepto la opción 5 que asciende notablemente, siendo esta la que más se repite en esta variable. Esto lo podemos observar en los cambios producidos en las medidas de tendencia central y dispersión, pues la media pasa de 2,67 a 4,22, y la moda de 2 a 5 . El motivo por el que asciende la desviación típica de 1,5 a 1,56 es porque se dispersan los datos con respecto a la media, que en el ejercicio inicial estaban más concentrados.

e. Presentación. El p-valor de esta variable es de 0,539, por lo que no constituye un dato significativo, ya que denota que no existen comportamientos diferentes entre el ejercicio inicial y el final. Por lo tanto, la hipótesis nula es una hipótesis de homogeneidad: la variable Presentación se distribuye igual antes de la implantación de los talleres de animación lectora que después de su realización.

Al distribuirse las frecuencias de forma más dispersa aumenta ligeramente la desviación típica en el ejercicio final, aunque la agrupación de los datos es muy similar a la del ejercicio inicial. Aparecen señaladas en el ejercicio casi todas las opciones porque cada uno de los alumnos presenta valores dispersos para la variable Presentación, por lo que para la interpretación es mejor atender a la distribución de las frecuencias. Se puede apreciar una tímida progresión, pues se produce un aumento 
de las frecuencias en la opción 5 (la mejor valorada para esta variable) y la media cambia del pretest al postest de 2,89 a 3,56.

f. Estilo. El p-valor en este caso es de 1, por lo tanto, no se trata de un dato significativo, ya que denota que no existen comportamientos diferentes entre el ejercicio inicial y el final. La hipótesis nula es una hipótesis de homogeneidad, es decir, la variable Estilo se distribuye igual antes de la implantación de los talleres de animación lectora que después de su realización.

Tal y como se puede observar de forma clara en la tabla de medidas de tendencia central y dispersión no se observa ningún cambio en la variable Estilo en el grupo experimental.

\section{Discusión de los Resultados y CONClusiones}

Tras el análisis estadístico de estas variables antes y después de la implantación del programa, se ha llegado a la conclusión de que los talleres de animación lectora que incluían actividades de escritura han logrado una ligera mejoría en la expresión escrita del alumnado, tal y como indican los cambios estadísticamente significativos en la variable Cohesión y los cambios positivos, aunque no estadísticamente significativos, de las variables Coherencia y Adecuación.

La propiedad textual que ha mejorado tras los diferentes talleres de animación lectora del programa que se ha realizado ha sido especialmente la cohesión. Se han mejorado, por lo tanto, aspectos como el uso de los diferentes campos léxicos, el empleo de las figuras de repetición, de los deícticos espaciales y temporales, la correcta utilización de enlaces, conectores y marcadores textuales o el uso adecuado de la puntuación. Son aspectos gramaticales, aunque las actividades relacionadas con la expresión escrita abarcaban todas y cada una de las propiedades textuales que se consideran en este estudio, las modificaciones positivas recaen sobre esta propiedad en concreto.

También se han producido ligeras mejorías que no tienen relevancia estadística en las propiedades de la coherencia y la adecuación. Se observan, en relación con la coherencia, cambios favorables en el contenido que se ofrece en los textos, en el orden de la exposición de las ideas, así como un mejor dominio de la estructura textual. Tal y como se indica en los resultados de Núñez, Fernández y Romero (2008), al mejorar la cohesión de los textos, también se produce una mejora de la coherencia, porque un texto bien cohesionado es un texto más coherente. A pesar de esto, los resultados para la variable Coherencia no son del todo significativos, porque el progreso de esta propiedad textual implica cambios también en otros aspectos como los mencionados anteriormente. Este es el motivo por el que el perfeccionamiento de esta variable no es tan contundente.

En cuanto a la adecuación, se intuyen discursos ligeramente más apropiados al contexto, y más claros en su propósito, aunque se mantiene el nivel de formalidad, que es escaso. El aumento de la puntuación para la variable Adecuación radica en dos aspectos. En primer lugar, que el alumnado aprendió durante la realización de los talleres de animación lectora a ser consciente del contexto en el que está inmerso en cada momento, y en segundo lugar adquirió pautas sistemáticas para adaptar sus escritos al contexto académico. Estos dos 
matices le han ayudado a asumir una serie de técnicas que adapta de forma sencilla a sus escritos para adecuarlos a la situación académica.

Sin embargo, el resto de las propiedades textuales -corrección, presentación y estilono han mostrado cambios significativos en los escritos del alumnado. Esto indica que son las variables estudiadas que presentan más dificultad para su adquisición en el proceso de enseñanza-aprendizaje del español como L2 y que, por lo tanto, es necesario establecer una línea de tareas diferentes que las refuercen. La variable Corrección era presumiblemente una de las de más difícil logro al tratarse de un ejercicio escrito en una segunda lengua; a pesar de que se establecieron actividades para mejorar esta propiedad en los talleres de animación lectora no se ha producido apenas avance, razón por la cual es importante cambiar la perspectiva desde la que se ha abordado en el transcurso del curso escolar. La variable Presentación podría parecer una propiedad que con unas técnicas básicas se podría haber adquirido, pero no ha sido así, hecho que se atribuye al contexto en el que vive el alumnado, el desinterés con el que a menudo se afrontan las actividades académicas, y al arraigo de algunas costumbres como la forma de presentar los trabajos de clase. Parece que el carácter lúdico del programa realizado en el aula, en este caso, no ha servido como elemento motivador.

Es especialmente significativo el estudio de la variable Estilo que, para todos los sujetos en ambos ejercicios, el inicial y el final, siempre ha obtenido la puntuación más baja según la escala empleada. Los escritos del alumnado carecen totalmente de estilo y los talleres de animación lectora no han mejorado esta situación, resultado, por otra parte, esperable. Probablemente el contexto en el que se ha realizado la investigación (alumnado extranjero), junto con el hecho de que el estilo requiere un cierto nivel de competencia escrita, sea la causa de este fenómeno. Son necesarios muchos cambios adicionales para que el alumnado asimile las características que debe tener un texto, oral o escrito, para que su estilo se considere aceptable. El cambio de esta percepción precisa de un camino complejo compuesto por conceptos y habilidades de diversos ámbitos, y que al final no es otro que una maduración de su alfabetización, proceso que en el contexto escolar no solo se puede abordar desde las áreas lingüísticas.

Coincidimos, por lo tanto, con Perdomo (2005), en que la metodología del taller de animación lectora mejora la calidad de la expresión escrita del alumnado; sin embargo, los resultados de su investigación fueron más favorables y las conclusiones acerca de la aplicación de esta herramienta con dicha finalidad, más positivas. La diferencia entre ambos estudios radica en el contexto y en la procedencia foránea del alumnado, hechos que pueden marcar la dificultad que tiene el alumnado inmigrante para mejorar las propiedades textuales sirviéndose únicamente del programa de animación lectora.

Estos resultados animan a utilizar los talleres de animación y los textos literarios como medios eficaces en las clases con alumnado inmigrante; se pueden plantear como alternativa metodológica aplicable en contextos de difícil desempeño como son los centros de compensación educativa, donde la motivación del alumnado a través de nuevas estrategias ha de ser el primer paso de un arduo pero gratificante trabajo. El alumnado de estos centros carece de competencia en la L2, de bagaje literario, así como de experiencias que aúnen literatura y diversión. Con esta propuesta se pretende acercar al alumnado a la literatura con una herramienta motivadora, dinámica, integradora y participativa para lograr una mejor competencia lingüística. Debido a la escasa motivación del alumnado y a que desde el ambiente 
familiar no se favorece el desarrollo de un entorno literario adecuado, estos adolescentes no perciben que sea una actividad útil para la vida real o de la que se pueda extraer un provecho tangible. Por ello, los talleres de animación lectora ayudan a materializar el hecho literario, haciéndolo más visible, aplicable a la vida real y, sobre todo, ayudan a establecer con él una relación más personal.

En cuanto a la expresión escrita, utilizar esta herramienta para su perfeccionamiento supone un cambio metodológico que ha de ser complementado con otros procedimientos de mayor duración que desarrollen esta destreza. Tal y como se ha observado en los resultados de la investigación, se necesitan otras actividades que contribuyan a desarrollar los aspectos más débiles de la expresión escrita del alumnado inmigrante, pero, aun así, se trata de una herramienta interesante que aporta un enfoque lúdico e integrador de la creación y la recepción literarias.

\section{Bibliografía}

Acosta Peñaloza, C., Rodríguez Pérez, E., Flórez Arcila, R., Jurado Valencia, F. (2013). ¿Lectores o leedores? Bogotá: Universidad Nacional de Colombia.

Álvarez-Álvarez, C. y Pascual-Díez. (2013). "Estudio de caso sobre la formación de lectores críticos mediante textos literarios en la Educación Primaria". Ocnos, 10: 27-53.

Cassany D.; Luna M.; Sanz G. (1997). Enseñar lengua. Barcelona: Graó.

Cassany, D. (2004). Construir la escritura. Barcelona: Paidós Ibérica.

Coseriu, E. (1987). Gramática, semántica y universales. Madrid.

Delmiro Coto, B. (2002). La escritura creativa en las aulas. En torno a los talleres literarios. Barcelona: Graó.

Fernández, C. y Fuentes, F. (1995). Curso de Estadística Descriptiva. Teoría y Práctica. Barcelona. Ariel.

Gómez-Villalba, E; Pérez González, J. y Maldonado, L. A. (1994): “Animación a la lectura y composición escrita". En Actas del VI Congreso Internacional de la Sociedad de Didáctica de la Lengua y la Literatura. Murcia, 193- 199.

Gómez-Villalba, E. et al. (2000): El desarrollo de las capacidades de los alumnos de educación primaria en el área de lengua castellana y literatura. Granada. GEU.

Gómez-Villalba, E.; Pérez González, J. (2001). "Animación a la lectura y comprensión lectora en educación primaria". Lenguaje y textos, 17: 9-20.

Gómez-Villalba, E.; Núñez, M. ${ }^{a}$ P. (2007). "La enseñanza de la lectura en el aula". Textos de didáctica de la Lengua y la Literatura, 44: 19-33.

Hernández Guerrero J. A. (2005). El arte de escribir. Barcelona: Ariel.

Junta de Andalucía (2006). Intervención coordinada de la Junta de Andalucía en zonas con necesidades de transformación social; aprobado por la Comisión Politica Andaluza. Sevilla: Servicio de Barriadas. Dirección General de Bienestar Social, Consejería para la Igualdad y Bienestar Social.

Moreno, V. (2007). "La escritura como estímulo de la lectura". Textos de didáctica de la Lengua $y$ de la Literatura, 44: 69-78.

Núñez, M. ${ }^{a}$ P., Fernández de Haro, E. y Romero, A. (2008). "Research in language didactics: a model for the evaluation of oral discursive competence in secondary education". Porta Linguarum. Revista Internacional de Didáctica de las Lenguas Extranjeras, 9, 111-126. 
Núñez, M. ${ }^{a}$ P., Fernández de Haro, E. y Romero, A. (2009). “Adquisición de habilidades metalingüísticas y enseñanza-aprendizaje de la composición escrita en educación primaria: reflexiones didácticas sobre los resultados de un estudio experimental". Porta Linguarum. Revista Internacional de Didáctica de las Lenguas Extranjeras, 12: 149-169.

Núñez, M. ${ }^{a}$ P.; Liébana, J.A. (2004). "Reflexión ética sobre la (des)igualdad en el acceso a la información". Comunicar, 22: 39-45.

Ong, W. (1982). Orality and Literacy. The Technologizing of the World. Londres: Methuen and Co.

Perdomo López, C. A. (2005). "La intervención específica en el proceso lector: repercusiones en la expresión escrita". Textos de Didáctica de la Lengua y de la Literatura, 39: 97-107.

Piñán, B. (2008). "Memoria literaria de la escuela: muros de tinta y papel" en Lomas, C. (coord.). Textos literarios y contextos escolares. La escuela en la literatura y la literatura en la escuela. Barcelona: Graó, 101-115.

Ramos García, A. M. ${ }^{\text {a }}$ (2010). "El reto de enseñar una lengua extranjera sin recursos". Porta Linguarum. Revista Internacional de Didáctica de las Lenguas Extranjeras, 10: 79-90.

Rodríguez Polo, J. (1999). Didáctica de la narración escrita: enseñanza del discurso narrativo en educación primaria. Granada: GEU. 